\title{
Abordaje biorregulador de Síndrome autoinflamatorio en un paciente pediátrico: Reporte de caso
}

\author{
Sandra Beltrán, ${ }^{1}$ Mariela Tavera, ${ }^{2}$ Sandra Palacios, ${ }^{3}$ Felipe Robayo, ${ }^{4}$ Carlos \\ Olmos, ${ }^{5}$ Oscar Tamayo, ${ }^{6}$ Fernando Zárate $^{7}$
}

Autor para correspondencia: Sandra Beltrán, sandrabeltranmd@gmail.com

${ }^{1}$ sandrabeltranmd@gmail.com

2 marielataveraz@gmail.com

${ }^{3}$ sandrapalacios950@hotmail.com

${ }^{4}$ felipero11@yahoo.com

${ }^{5}$ colmos.8600@gmail.com

6 oscartamayo.ot@gmail.com

${ }^{7}$ zaratefer@hotmail.com

\section{Resumen}

Se presenta escolar con fiebre recurrente asociada a manifestaciones cutáneas y dolores articulares sin retraso pondoestatural, con respuesta inflamatoria durante los episodios, cor diagnóstico de síndrome autoinflamatorio multifactorial tipo SURF (Syndrome of Undifferentiated Recurrent Fever), o uSAIDS (Enfermedad Autoinflamatoria Sistémica Indefinida) sin demostración de mutaciones clásicas, con una carga epigenética por antecedentes familiares sin respuesta a manejo usual con antipiréticos, antiinflamatorios y corticoides, quien respondió de forma beneficiosa a terapia biorreguladora vs curso natural de la enfermedad autoinflamatoria no clásica. La Medicina Biorreguladora de Sistemas (MBS) por tratarse de un paciente pediátrico se administró por vía nebulizada y nasal por 8 semanas, con lo que se demostró mejoría en la disminución de la fiebre en $80 \%$ y las lesiones cutáneas en un $65 \%$, con la consecuente mejoría en la calidad de vida del paciente y su familia.

Palabras clave: case report, Recurrent Fever, Periodic fever, autoinflammatory syndrome, SURF, ultra low doses, integrative medicine 


\section{Introducción}

Los Síndromes de Fiebre Periódica conocidos también como Enfermedades Autoinflamatorias Sistémicas (SAIDs, por sus siglas en inglés Systemic Autoinflammatory Diseases), se caracterizan por episodios de fiebre con manifestaciones variables tales como serositis, rash, linfadenopatías, artritis asociadas a reactantes de fase aguda elevados (1) y, que pueden comprometer la piel en forma inespecífica, sin que se encuentre evidencia de autoinmunidad o alteración de la respuesta inmune adaptativa. Estas enfermedades autoinflamatorias comparten una respuesta inflamatoria hiperactiva, dada por trastornos caracterizados por la disregulación del sistema inmune innato a señales de peligro endógenas o exógenas, ya sean asociadas a alteraciones del IL-1B, Interferón tipo I y Factor Nuclear -kB (FN-қB), activación de inflamasomas, así como otros factores contribuyentes monogénicos o multifactoriales como son autofagia, epigenética, disregulación del microbioma y autoanticuerpos, entre otros (2). Hasta ahora van más de 30 enfermedades febriles recurrentes monogénicas descritas, pero al menos el $80 \%$ de los pacientes no tienen un diagnóstico genético claro, y a pesar de que el estudio genético es un factor clave en la evaluación clínica de pacientes con trastornos autoinflamatorios, no todas las variantes tienen correlación genotipo-fenotipo conocidas y permanecen aún sin diagnóstico molecular o genético (3)(4)(1).

Actualmente, se considera que al menos entre el $40-60 \%$ de los pacientes cursan con fenotipos típicos de SAIDs, sin embargo aquellos que no cumplen con los criterios se agrupan en Enfermedad Autoinflamatoria Sistémica Indefinida (uSAIDs), también conocida como SURF (Syndrome of Undifferentiated Recurrent Fever por las siglas en inglés), en donde se plantean orígenes multifactoriales, poligénicos con influencias ambientales que modulan el fenotipo (4)(2). Muchos de los SAIDs pueden mostrar síntomas superpuestos, parciales o atípicos, incluyendo lesiones cutáneas de diferentes tipos (lesiones urticarianas, erupciones maculopapulares, pústulas, úlceras, granulomas y erisipela-like), sin embargo ninguna de ellas es específica para alguna enfermedad por lo que el diagnóstico diferencial es extremadamente difícil y por lo tanto dependiente de pruebas genéticas, que con la utilización de secuenciación de última generación han optimizado el diagnóstico de estas patologías no caracterizadas en forma importante. Hasta la fecha se han registrado más de 800 variantes genéticas y se 
está actualizando continuamente la guía para clasificación de patogenicidad y correlación de las mismas (5)

En una cohorte seguida en el Rady Children's Hospital en San Diego, se observó que hasta el $70 \%$ de los pacientes no cumplían criterios de SAIDs monogénicos ni poligénicos. Se encontró que el $61 \%$ correspondían al sexo femenino, con edad promedio de inicio de la enfermedad de 2.7 años, con episodios febriles de duración entre 10 a 68 días con un promedio de 30 días y además, de los síntomas autoinflamatorios clásicos se encontraban inmunodeficiencias y alergias. No se cuenta con información en nuestro medio de esta condición, sin embargo, con frecuencia cada vez mayor nos enfrentamos en nuestra práctica diaria este tipo de pacientes (1)

El tratamiento de los pacientes con SAIDs va encaminado a suprimir la inflamación sistémica. El uso de corticoides durante los episodios pueden ser una opción en algunos pacientes e incluso a veces se requieren dosis mayores en la medida que la enfermedad progresa. Otros tratamientos se basan en terapia dirigida a citoquinas, tales como antagonistas del receptor de IL-1 (Anakinra, Canakinumab y Rilonacept), los cuales tienen perfiles de seguridad similares, con un mayor riesgo de infecciones bacterianas no oportunistas. Igualmente se han usado moléculas inhibidoras de la autoinflamación como colchicina con buenos resultados pero mayores efectos adversos gastrointestinales. En los pacientes en que esta terapéutica falla, debe valorarse el uso de anti IL-1 ya mencionados, drogas anti FNT (Etanercept), anti IL-6 como el Tocilizumab e inhibidores de la Janus-Kinasa como el Tofacitinib, los cuales pueden reducir la actividad de la enfermedad (3)(2)(6). Las terapias no dirigidas son ineficaces o, en algunos casos, pueden incluso empeorar el cuadro.

Para pacientes con uSAIDS no se dispone de ningún fármaco específico. Actualmente el tratamiento consiste en inmunosupresión inespecífica con corticosteroides y otros fármacos antirreumáticos modificadores de la enfermedad, y dados los arquetipos de medicamentos de baja dosis se pensó en la administración de medicina integrativa o Medicina Bioreguladora de Sistemas (MBS) con el objetivo de inmunomodular su respuesta inflamatoria y 
obtener una homeodinamia así como un mejor funcionamiento de su red mitocondrial. Igualmente se conoce que desde el inicio de los síntomas hasta el diagnóstico puede pasar en promedio hasta 7.3 años por lo que es indiscutible la necesidad de identificar nuevos objetivos de tratamiento y diagnóstico, los cuales pueden ir enfocados a identificar e incidir en mecanismos ya mencionados como autofagia, metilación del ADN, expresión de microARN, composición del microbioma e inflamación de bajo grado y formación de autoanticuerpos entre otros(2).

\section{Descripción del caso}

Se presenta paciente femenina de 5 años de edad, quien consulta por primera vez a los 24 meses, por cuadro de fiebre recurrente desde el año de edad, cuantificada hasta $39^{\circ} \mathrm{C}$, con periodicidad inicial de 4 horas y posteriormente más espaciada, con duración promedio del episodio de 4 hasta 20 días y con una recurrencia entre 30 a 45 días, tornándose más frecuente a partir de los 4 años de vida, de predominio vespertino y nocturno manejadas con acetaminofén. A los 2 años de edad presenta episodio febril con diagnóstico de otitis media aguda y laringitis, recibiendo acetaminofén, ibuprofeno, amoxicilina y Bacillus claussi. En otro episodio febril prolongado recibe prueba terapéutica con prednisolona sin modificar su curso y presentación. La fiebre se acompaña en algunas ocasiones de dolor en manos y en los últimos meses dolor en los pies, y a partir de los 2 años y medio en algunos de dichos episodios recurrentes, la fiebre se acompaña de lesiones urticariformes circinadas, numulares, induradas, eritematovioláceas, edematosas, calientes al tacto, dolorosas a la digitopresión, evanescentes y de pocos milímetros de diámetro hasta $1 \mathrm{~cm}$ diseminadas en cara y cuerpo, con predominio en miembros inferiores con duración promedio de 4 días y que dejan pigmentación residual. Los episodios febriles se acompañan de hiperactividad y logorrea con lenguaje coherente e irritabilidad. No se evidencia retraso pondoestatural, y se detectaron ocasionales adenopatías cervicales no patológicas, en alguna ocasión vaginitis y lesiones eritematosas perianales que duraron hasta 3 meses. 
Antecedentes de importancia: madre con endometriosis y ovarios poliquísticos que requirió inductores de ovulación, embarazo que cursó con Infección de vías urinarias manejada con antibiótico y parto por cesárea por iterativa. Historia en familia materna de enfermedades autoinmunes como LES y artritis reumatoidea, y Diabetes en familia paterna y materna. Lactancia materna hasta primer mes de vida, con intolerancia a diferentes fórmulas infantiles, manifestadas con reflujo gastroesofágico y dermatitis de contacto irritativa perianal por un lapso de 3 meses. Al año y medio se detecta alergia a la clara de huevo y al maní. Cuadros virales como Influenza tipo A y Adenovirus a los 23 y 31 meses, y Covid-19 a los 4 años y medio. Exposición a hongos y humedad ambiental al año de edad, que concuerda con aparición de la sintomatología. Vacunación completa para la edad. Ingiere leche de vaca.

Por la periodicidad de las fiebres y la no evidencia de focos infecciosos asociados en la mayoría de los episodios febriles, así como la presencia de lesiones urticariformes asociadas a los hallazgos de laboratorio descritos en la Tabla 1, se plantea diagnóstico de Síndrome de fiebres periódicas o SAIDs, por lo que se decide ampliar estudios con biopsia de piel que reporta: "Discreto edema en dermis superior asociado a infiltrado inflamatorio tipo linfoide perivascular y algo perianexial con ocasionales neutrófilos entremezclados, con hallazgos de inmuno directa que muestran lgG++ y fibrinógeno ++ perivascular superficial. IgA, IgM, C1q, C3 y C4 negativos, cambios compatibles con Síndrome Autoinflamatorio urticarial. Se realiza estudio genético de Exoma de 36 genes para fiebre periódica y secuenciación de mutaciones para deficiencia de Mevalonatokinasa que son negativos. Estos hallazgos no conclusivos para una entidad específica de SAIDs consolidaron el diagnóstico de Enfermedad Autoinflamatoria Sistémica Indefinida (uSAIDs).

Desde el punto de vista terapéutico, además de la terapia convencional ya mencionada, las lesiones en piel se manejaron inicialmente con antihistamínicos con poca respuesta y desde los 3 años se administra Engystol@ y Traumeel® orales, los cuales suspenden al año ante la no evidencia de beneficio terapéutico. Debido a la falta de resultados a las terapias previas y a la persistencia de los episodios febriles y lesiones en piel concomitantes con aparición de otros síntomas y signos como cefalea, irritabilidad, dolores en manos y pies, tos seca, 
estreñimiento, enuresis diurna y nocturna y la presencia de su diátesis atópica, se decide recurrir a MBS a los 4 años y 9 meses de edad con nebulizaciones semanales con Ubichinon compositum $\AA$, Lymphomyosot $\AA$, Nuxeel Homaccord $\AA$ y Berberis Hommaccord® ( 1 ampolla de cada uno) y aspersión nasal en cada fosa 3 veces al día con una mezcla de Coenzyme compositum $\AA$, Traumel® y Engystolß (1 ampolla de cada uno) por 8 semanas, con notoria disminución de los síntomas por lo que nunca necesito el uso de colchicina a pesar de haber sido prescrita. (Ver Tabla 2).

\section{Punto de vista del paciente}

Según la madre el cambio más notorio fueron los "eritemas", evidente a la semana del inicio de la terapia biorreguladora. "Si lo pusiera en cifras, ha mejorado un 50\% en fiebre y un 70- 80\% en eritemas"; además refiere que fue fácil la nebulización y la aplicación de medicamentos nasales.

\section{Discusión}

Nuestra paciente presenta múltiples factores caracterizados como exposoma que llevan a inflamación crónica causando disregulación en diferentes redes del organismo tales como, la exposición in útero a homotoxinas generadas por estrés, inflamación crónica materna (Ovario poliquístico), químicas (Inductores de la ovulación en la madre por muchos años), así como cambios epigenéticos dados por los antecedentes maternos y familiares de Ovario poliquístico y enfermedades autoinmunes donde se han demostrado alteraciones en la metilación y desmetilación del ADN, lo que puede conducir a la activación de genes del inflamosoma y la producción de citoquinas proinflamatorias en la enfermedad autoinflamatoria (7) y reprogramación neuroendocrina descrita en los fetos de madres con ovario poliquístico (8), que presentan niveles elevados de andrógenos e insulina que afectan el funcionamiento endocrino-metabólico. De la misma forma se destacan alteraciones en la red de mucosas/piel así como en su microbiota, la cual se adquiere desde la etapa prenatal y es modificada por factores ambientales perinatales y postnatales. En este caso en particular, se refleja en la exposición a antibióticos in útero y postnatal, parto por cesárea, fórmulas lácteas, cambios 
dietéticos, entre otros, causando disbiosis, jugando un papel central en los orígenes y perpetuación de las enfermedades inflamatorias crónicas (9). La biopsia, aunque puede proporcionar información adicional, no es patognomónica ya que tanto el grado de edema y el componente principal en los infiltrados pueden variar en las distintas patologías (4).

Se evidencio afección de la red neuroendocrina, dada por manifestaciones neuromusculares, fatiga e irritabilidad, las cuales se exacerbaron posterior a la infección por SARS CoV2/Covid-19.

En la inflamación persistente, las toxinas se depositan en la matriz manteniéndose en acidosis tisular y bloqueando la actividad enzimática de los organelos de las células, lo que activa mecanismos del sistema inmune innato, encargados de regular la inflamación generada por el acúmulo de células con alteración mitocondrial descritas en SAIDs (1)(2).

Analizando los medicamentos biorreguladores con los que se obtuvo respuesta clínica encontramos que el Ubichinon compositum® actuó desde sus múltiples componentes estimulando a los sistemas enzimáticos no funcionales los cuales están presentes a partir de las fases de deposición e impregnación de la enfermedad, gracias a la composición de quinonas y otros factores metabólicos biorreguladores, minerales y plantas. Uno de sus componentes es el colchicum autumnale que disminuye el daño enzimático de manera específica por despolimerización. El Coenzyme compositum® estimula los sistemas enzimáticos defectuosos en las fases matricial y celular, a través de los intermediarios y cofactores del ciclo de Krebs que son antioxidantes y detoxificantes. Estos dos productos mejoran la oxigenación a través de la membrana mitocondrial y activa a nivel de la mitocondria la primera línea de defensa en la infección viral. Por otro lado, el Lymphomyosot® favorece el drenaje linfático y la respuesta inmune específica principalmente a nivel del MALT(Tejido Linfoide Asociado a Mucosas) y del SALT (Tejido Linfoide Asociado a piel), facilitando la movilización de toxinas desde la matriz extracelular y disminuyendo la hipoxia tisular y la acidificación, mejorando así el metabolismo del tejido conjuntivo. 
el impacto terapéutico respecto al funcionamiento mitocondrial y la autorregulación de redes comprometidas, toda vez que las interleucinas y los parámetros de reactantes de fase aguda son inespecíficos, y no contamos con patrones moleculares para correlacionar con la evolución y mejoría clínica que presentó nuestra paciente con MBS.

\section{Conclusión}

Este caso ilustra no solo el reto diario al que se enfrentan los clínicos que evalúan pacientes con síndromes febriles recurrentes que no cumplen criterios de cuadro clásicos monogénicos ni poligénicos como el Síndrome PFAPA (Fiebre Periódica, Estomatitis Aftosa, Faringitis y Adenitis cervical) y que comparte con el SURF, además de su curso benigno hacia la mejoría con el tiempo, la ausencia de impacto en el desarrollo pondoestatural y los marcadores inflamatorios intermitentes, el abordaje terapéutico donde aún se encuentran terrenos por explorar como lo es la MBS, cambios en la dieta, drenaje de la matriz y autorregulación de la actividad mitocondrial y de otras redes comprometidas, buscando la resolución de la enfermedad.

En este paciente se demostró mejoría con MBS cuantificada para la fiebre en un $80 \%$ (en intensidad y frecuencia) y para las lesiones cutáneas en un $65 \%$. A la fecha, no fue posible que la paciente se tomara los laboratorios ordenados posteriores al inicio del tratamiento, pero estamos a la espera para completar la correlación paraclínica.

Esto deja abierta una ventana de investigación desde el enfoque terapéutico no convencional para pacientes con fiebres recurrentes y enfermedades autoinflamatorias sistémicas hasta ahora no explorado y que amerita estudios clínicos y moleculares a la par que avanza el conocimiento de la correlación genética con los diferentes fenotipos en uSAIDS. 


\section{Agradecimientos}

Agradecemos a Laboratorios Heel, al Dr. Baltazar Guerrero por su guía y orientación y a todos nuestros profesores del Diplomado quienes nos aportaron sus conocimientos y experiencia para sembrar en nosotros la forma de abordar a nuestros pacientes de una manera más holística. 


\section{$\underline{\text { Referencias }}$}

1. Broderick L, Hoffman HM. Pediatric recurrent fever and autoinflammation from the perspective of an allergist/immunologist. $\mathrm{J}$ Allergy Clin Immunol [Internet]. 2020;146(5):960-966.e2. Available from: https://doi.org/10.1016/j.jaci.2020.09.019

2. Krainer J, Siebenhandl S, Weinhäusel A. Systemic autoinflammatory diseases. J Autoimmun. 2020;109(January).

3. Broderick L. Hereditary Autoinflammatory Disorders: Recognition and Treatment. Immunol Allergy Clin North Am [Internet]. 2019;39(1):13-29. Available from: https://doi.org/10.1016/j.iac.2018.08.004

4. Luu I, Nation J, Page N, Carvalho D, Magit A, Jiang W, et al. Undifferentiated recurrent fevers in pediatrics are clinically distinct from PFAPA syndrome but retain an IL-1 signature. Clin Immunol [Internet]. 2021;226(February):108697. Available from: https://doi.org/10.1016/j.clim.2021.108697

5. Rowczenio DM, Lachmann HJ. How to prescribe a genetic test for the diagnosis of autoinflammatory diseases? Press Medicale [Internet]. 2019;48(1):e49-59. Available from: https://doi.org/10.1016/j.lpm.2018.08.015

6. Nigrovic PA, Lee PY, Hoffman HM. Monogenic autoinflammatory disorders: Conceptual overview, phenotype, and clinical approach. J Allergy Clin Immunol [Internet]. 2020;146(5):925-37. Available from: https://doi.org/10.1016/j.jaci.2020.08.017

7. Reumatología RC De, María A, Lima $T$, Yolanda A, Torres R. Epigenética y enfermedades autoinmunes sistémicas. Rev Cuba Reumatol. 2015;17(3):216-21.

8. Concha Francisca, Sir Teresa, Recabarren S PF. Epigenética del síndrome de ovario poliquístico. Rev Med Chile. 2017;145:907-15. 
9. Renz H, Holt PG, Inouye M, Logan AC, Prescott SL, Sly PD. An exposome perspective: Early-life events and immune development in a changing world. J Allergy Clin Immunol [Internet]. 2017;140(1):24-40. Available from: http://dx.doi.org/10.1016/j.jaci.2017.05.015

\section{Archivos adjuntos}


CORRELACIÓN FIEBRE CON LESIONES CUTÁNEAS

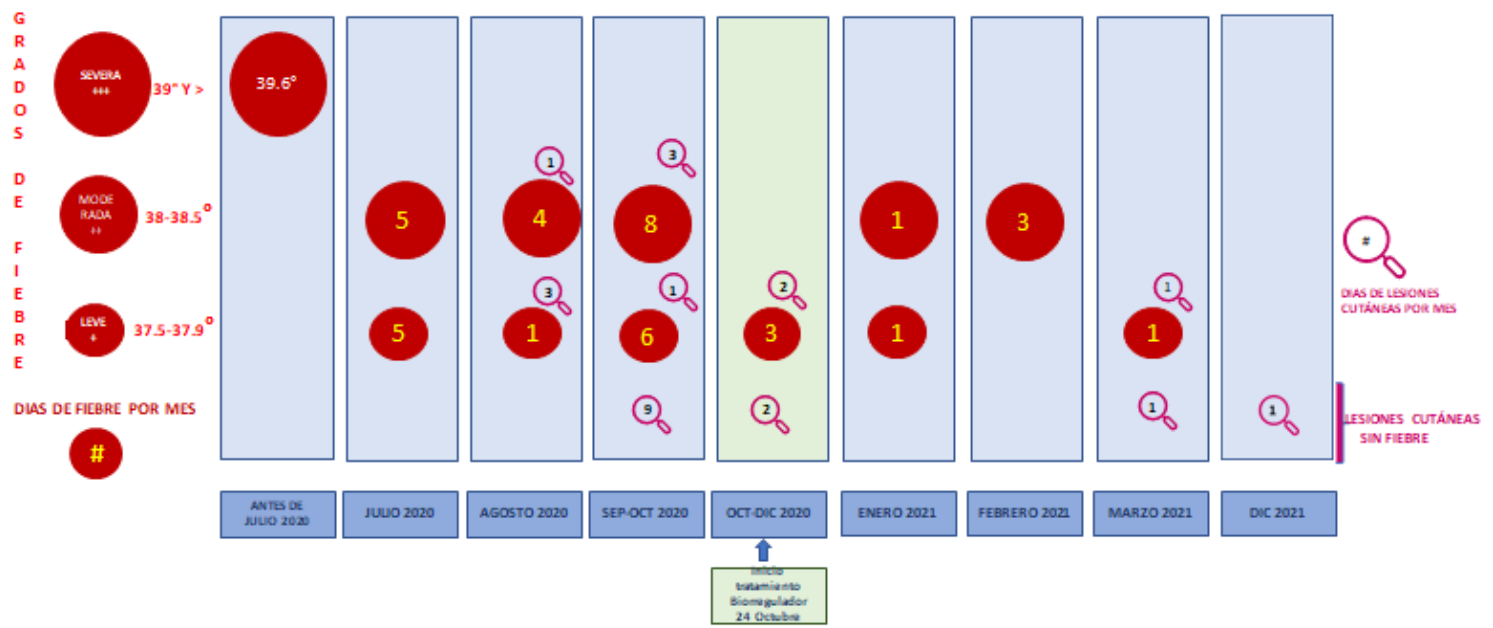

Se observa como la fiebre disminuye posterior al inicio de medicina biorreguladora, tanto en intensidad como en frecuencia en $80 \%$. Las lesiones cutáneas disminuyen en número y duración en un $65 \%$, observándose incluso en ausencia de fiebre.

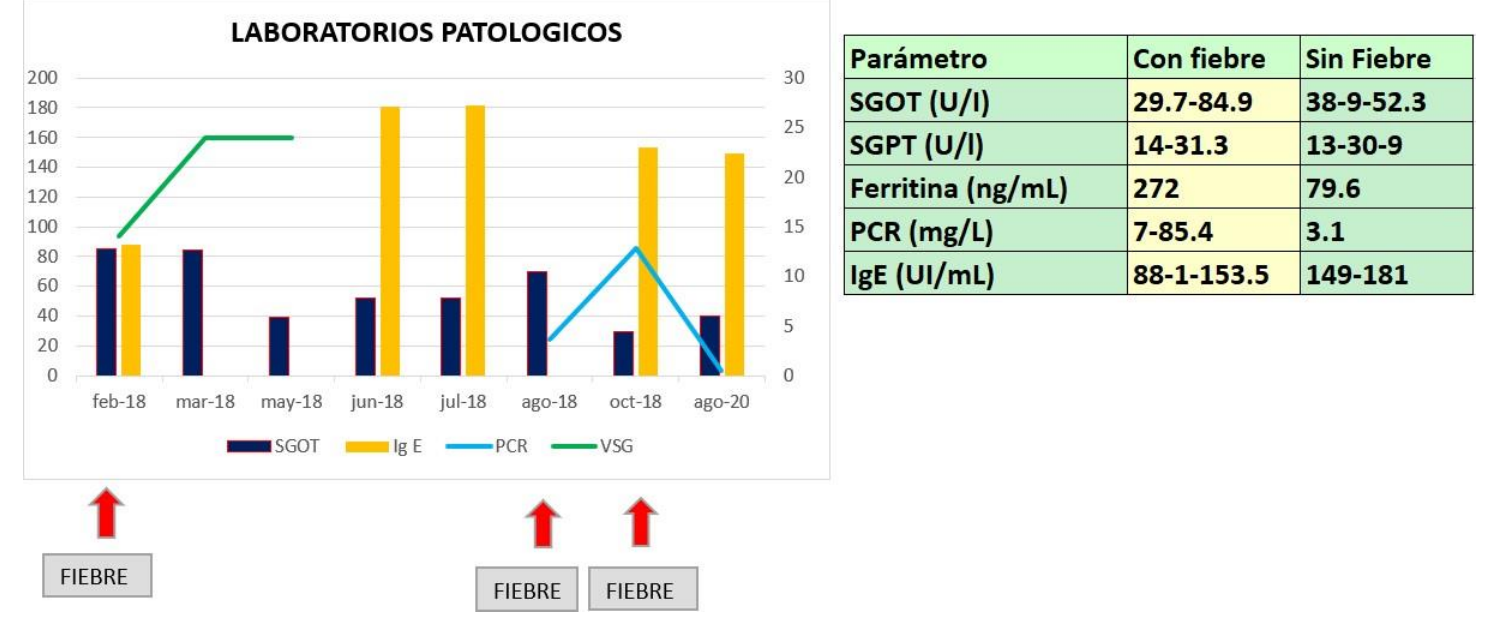

Tabla 1. Laboratorios Patológicos. Además de la elevación de PCR con normalidad en intervalos sin fiebre; hay discreto aumento de la VSG durante episodios febriles. Se encuentra leucocitosis con neutrofilia en un episodio febril, en los restantes linfocitosis. IgD elevada(> $100 \mathrm{UI} / \mathrm{ml}$ ) cerca a un episodio febril; y otro valor normal sin fiebre. IgG y A por encima del rango aunque oscilantes. IgE elevada compatible con fondo atópico. 